\title{
THE RUIN PROBLEM WITH A FINITE TIME HORIZON*
}

\author{
By Marc-Henri AmSLer \\ University of Lausanne, Switzerland
}

\begin{abstract}
The paper presents an extension of the classical Cramér-Lundberg ruin theory: the famous upper bound for the ruin probability with an infinite time horizon can be extended in a certain sense to the short and middle term case. Furthermore, a relation between the average values of lifetime and ruin amount is given.
\end{abstract}

\section{KEYWORDS}

Ruin theory, middle term horizon, lifetime, ruin amount.

\section{INTRODUCTION}

In order to assess the financial stability of an insurance portfolio, one usually utilizes the notion of "mathematical ruin". Ruin being the phenomenon by which a portfolio passes from the state "to be" to the state "to be no longer", actuaries have naturally sought to measure the danger of such a passage by its "probability". Numerous studies have unfortunately shown that the notion of "ruin probability" is not easy to handle, in theory as well as in practice. This difficulty, and it seems to be a major one, requires the search for another quantifier of the notion of ruin than that of probability.

The present article recalls firstly the notion of the ruin "counter-utility", proposed elsewhere, and which represents a more elaborate measure of danger than that of "probability". The ruin counter-utility takes into account three characteristics of ruin, that is:

the probability of its occurrence

the size of the ruin amount

the time of its occurrence.

The counter-utility is the greater, the larger the ruin amount, and is the smaller the more distant the event. The notion of counter-utility depends very closely on that of utility; in a certain way it reverses its properties.

Secondly, the article shows that the celebrated upper bound of the ruin probability, indicated by Lundberg, valid in an infinite time horizon, can be generalized to the case of a finite time horizon. For this purpose the future should not be separated in two distinct periods, the considered period, and the one left out, but should be considered in its totality with a progressive attenuation

* Paper presented to the 16th ASTIN Colloquium, Liège, September 1982

ASTIN BULLETIN Vol. 14, No. 1 
of what occurs, by a phenomenon similar to fog, limiting vision to a certain horizon (time-stumping phenomenon).

By means of this new approach to the ruin problem it is easier to acquire, without mathematical complications, some knowledge of the seriousness of ruin in a limited time horizon.

The considered portfolios will be characterised by the following symbols:

$X \quad$ aggregate claim amount

$f(x) \quad$ density function of $X$

$M(a)$ moment generating function of $X$

$P \quad$ total risk premium per annum of the portfolio

$P_{E} \quad$ Esscher premium of the portfolio

$R_{t} \quad$ risk reserve, at time $t$

$T$ time elapsed until the first ruin

$q_{t} \quad$ probability of the first ruin at time $t$

$\psi \quad$ ruin probability in the future

$Z \quad$ amount of the first ruin

$Z_{t} \quad$ amount of the first ruin, at time $t$

$g_{t}(z)$ density function of $Z_{t}$

$u(x) \quad$ utility function

$\bar{u}(x) \quad$ counter-utility function

$\bar{U}(Z) \quad$ ruin counter-utility

a risk aversion coefficient

$b \quad$ time stumping coefficient

$\theta \quad$ time horizon

The article considers, for means of simplification, portfolios that are stationary in time and create independent total claim amounts, and is based on exponential utility and counter-utility functions. Under these assumptions, the results are valid for an arbitrary process, not necessarily Poisson.

\section{CLASSICAL RESULTS OF THE RUIN THEORY}

The classical ruin theory is dominated by two notions: security margins and ruin probability. Here are some known properties:

\section{Security Margins}

The zero utility principle

$$
U\left(R_{t+1}\right)=\int u\left(R_{t}+P-x\right) \cdot f(x) \cdot d x,
$$

under the hypothesis of an exponential utility function $u(x)$, leads to the following formula for the premium $P$, margin included:

$$
e^{a P}=M(a)
$$


where

$$
M(a)=\int e^{a x} \cdot f(x) \cdot d x
$$

which is equivalent to

$$
P=\frac{1}{a} \ln M(a)
$$

\section{Ruin Probability}

The ruin probability (first ruin, with $t$ an integer)

$$
\psi=\sum_{t=1}^{\infty} \int_{0}^{\infty} g_{t}(z) \cdot d t
$$

is limited by Lundberg's upper bound

$$
\psi<e^{-a \cdot R_{0}}
$$

The coefficients $a$ in (2) and (4) are identical.

\section{THE NOTION OF COUNTER-UTILITY}

The notion of utility is borrowed from economics: it allows the determination of preferences between many situations.

The notion of counter-utility is derived from that of utility; it adds, for insurance purposes, a possibility to measure singularity considered situations.

Let $Y$ be a random variable. The expression

$$
\bar{U}(Y)=\int \bar{u}(y) \cdot f(y) \cdot d y
$$

in which the function $\bar{u}(y)$ satisfies

$$
\bar{u}(y)>0 ; \quad \bar{u}^{\prime}(y)>0, \quad \bar{u}^{\prime \prime}(y) \geqslant 0
$$

is called the counter-utility of $Y$. The function $\bar{u}(y)$ is the counter-utility function.

It is to be noted that the requirement $\bar{u}^{\prime \prime} \geqslant 0$ is the reverse of $u^{\prime \prime} \leqslant 0$, which the utility function is subjected to. $\bar{U}(Y)$ can be used to measure a risk: in $\bar{U}(Y)$, the big values of $Y$ are weighted overproportionally.

The exponential function

$$
\bar{u}(y)=e^{a y}
$$

satisfies our exigences. The coefficient $a$ is called the risk aversion coefficient.

The relation (1) expresses that, on the basis of an exponential counter-utility function, there is equivalence between the counter-utility of the premium $P$ (left-hand term) and that of risk $X$ (right-hand term):

$$
e^{a P}=M(a) \text {. }
$$


This last relation formalises the "counter-utility equivalence principle between premium and risk". Through this interpretation of (1), the notion of counterutility replaces that of utility and the counter-utility equivalence principle that of the zero utility principle.

\section{The Counter-Utility of Risk}

In order to estimate the risk situation of a portfolio, we consider the risk reserve, more exactly the value

$$
\bar{R}_{t}=-R_{t}
$$

which is representative of the danger (a positive danger if the risk reserve is negative and inversely), the counter-utility of this $\bar{R}_{t}$ is, at time $t$ and for an exponential counter-utility function:

$$
\bar{U}\left(\bar{R}_{t}\right)=\int e^{a \bar{r}} \cdot f_{t}(\bar{r}) \cdot d \bar{r} .
$$

For $t=0$, the risk reserve has a known value; therefore

$$
\bar{U}\left(\bar{R}_{0}\right)=e^{a \bar{R}_{0}}=e^{-a \cdot R_{0}} .
$$

If the premiums are determined by the zero utility principle, or by the counterutility equivalence principle, it can be easily shown that the counter-utility of the risk situation is constant in time:

$$
\bar{U}\left(\bar{R}_{t}\right)=\bar{U}\left(\bar{R}_{0}\right) \quad t=1,2,3, \ldots
$$

therefore

$$
\bar{U}\left(\overline{\boldsymbol{R}}_{t}\right)=e^{-a \cdot R_{\mathrm{n}}} .
$$

The value of Lundberg's upper bound (4) of the ruin probability $\psi$ is thus equal to the counter-utility of the risk situation of the portfolio at the beginning of time, and, because of the constancy of this counter-utility in time, equal to the counter-utility of the risk situation at time $t$ (always under the hypothesis of a counter-utility equivalence between premium and risk).

\section{The Counter-Utility of Ruin}

If $Z_{t}$ represents the ruin amount (first ruin), at time $t$, it can be shown without difficulty that the counter-utility of the ruin situation for all future years, generalizing (3):

$$
\bar{U}(Z)=\sum_{t=1}^{\infty} \int_{0}^{\infty} e^{e z} \cdot g_{t}(z) \cdot d z
$$

is equal to the value of the counter-utility of the risk situation:

$$
\bar{U}(\boldsymbol{Z})=\bar{U}\left(\overline{\boldsymbol{R}}_{t}\right)=\bar{U}\left(\overline{\boldsymbol{R}}_{0}\right)=e^{-a \cdot \boldsymbol{R}_{\mathrm{o}}} .
$$


Thus the counter-utility $\bar{U}(Z)$ of the ruin situation for the entire future, the counter-utility $\bar{U}\left(\overline{\boldsymbol{R}}_{t}\right)$ of the risk situation at $t$, notably when $t=0$, and the upper bound of the ruin probability according to Lundberg are identical.

\section{INCREASING COUNTER-UTILITY PRINCIPLE}

The formulae and properties stated so far are known.

The greater part of the following is new. The model referred to above can be generalized (always under the hypothesis of a stationary process and of an exponential utility function) in view of studying the equilibrium and the ruin conditions in the short and medium term.

\section{The Counter-Utility of Risk}

In reality, for a given aversion coefficient, premium $P$ and risk $X$ are not entirely equivalent. The relation (1) opens up three cases

$$
e^{a P} \geqq M(a)
$$

corresponding successively to an over-taxed premium, a premium equivalent in counter-utility and an under-taxed premium. We transform this last relation into an equation by the introduction of a supplementary factor

$$
e^{a P}=M(a) \cdot e^{-b} .
$$

The coefficient $b$ measures the level of under-taxation of risk $X$ by premium $P$. The coefficient $b$ is positive in the case of under-taxation, which we will deal with later. Under these conditions, it can be easily shown that the counter-utility of the risk situation is no longer constant in time, but evolves as follows:

$$
\bar{U}\left(\bar{R}_{t+1}\right)=\bar{U}\left(\bar{R}_{t}\right) \cdot e^{b} .
$$

Given the initial value of $\bar{U}\left(\bar{R}_{0}\right)$ according to (8), we have

$$
\bar{U}\left(\overline{\boldsymbol{R}}_{t}\right)=e^{-a R_{0}+b t}
$$

which generalises (6).

An under-taxed premium $(b>0)$ leads therefore to an increase of the risk counter-utility, an over-taxed premium $(b<0)$ to a decrease.

The recurrent relation (10) defines the increasing counter-utility principle (or decreasing if $b<0$ ).

The evolution of a portfolio with a constant counter-utility, seen under point 3 by the application of the zero utility principle, corresponds to the limit case $b=0$ between the two cases $b>0$ and $b<0$.

Formula (10) has an undoubtedly intuitive meaning.

\section{The Counter-Utility of Ruin}

In the case of an under-taxed portfolio (related to the counter-utility equivalence principle) it can be shown that if the definition (7) of the ruin counter-utility is 
generalized by the introduction of the factor $e^{-b}$, such that

$$
\bar{U}(Z)=\sum_{t=1}^{\infty} e^{-b \cdot t} \cdot \int_{0}^{\infty} e^{a \cdot z} \cdot g_{t}(z) \cdot d z
$$

then the ruin counter-utility keeps its standard value (8)

$$
\bar{U}(Z)=e^{-a \cdot R_{0}}
$$

In expression (12), the coefficients $a$ and $b$ are bound by relation (9). The introduction of the factor $e^{-b \cdot t}$ in (12) has the following meaning. The factor $e^{-b t}(b>0)$ reduces the weight of future ruins in $\bar{U}(Z)$ : the more distant the ruin the greater the reduction of $\bar{U}(Z)$. This corresponds to a "time stumping" phenomenon. The coefficient $b$ is the time stumping coefficient and $e^{-b}$ the stumping factor.

For an aversion coefficient leading to the equivalence in counter-utility between premium and risk, the stumping coefficient $b$ vanishes and (12) is identical to (7).

The expression $e^{-a \cdot R_{0}}$ according to (13) is thus a practical measure of the risk situation of a portfolio: it takes into account by means of the risk coefficient $a$ the size of the ruin amount, and by means of the stumping coefficient $b$, the imminence of the ruin. The notion of ruin counter-utility (12) can thus be used to measure the financial equilibrium of an insurance portfolio. This notion is more elaborate than that of ruin probability, which only considers the alternative "to be or to be no longer".

\section{FINITE TIME HORIZON}

A second interpretation of formula (12) leads to an estimation of the risk situation of a portfolio limited to a finite time horizon.

If, in expression (12), we replace the ruin counter-utility at $t$, that is

$$
\int_{0}^{\infty} e^{a z} \cdot g_{t}(z) \cdot d z
$$

by the length of the period (1 year) during which the said ruin might occur, expression (12) becomes

$$
\sum_{t=1}^{\infty} e^{-b t} \cdot 1 \quad(b>0)
$$

whose signification is that of the future (up to infinity) subjected to the stumping process mentioned above.

Let us designate by $\theta$ this value, which we will call the "time horizon". Because

$$
\sum_{t=1}^{\infty} e^{-b t}=\frac{1}{e^{b}-1}
$$


we find for the period $\theta$ :

$$
\theta=\frac{1}{e^{b}-1}
$$

or inversely

$$
e^{b}=\frac{\theta+1}{\theta}
$$

The greater the stumping coefficient $b$ the shorter the horizon; this is a natural property of a stumping phenomenon.

If one accepts the notion of "time horizon", then expression (12) measures the ruin counter-utility in a "finite time horizon $\theta$ ". The interpretation of expression (12) by means of the time horizon allows us to formulate an extension of the Cramér-Lundberg's theory when considering the short and medium term. The formula considers the entire future until infinity, but reduces the "weight" of future events in function of their distance in time, just as the discount phenomenon with regards to payments in a distant future.

\section{RUIN AMOUNT AND PORTFOLIO LIFETIME}

The method used above to estimate the financial equilibrium of insurance portfolios allows developments in various directions. Here follows what can be deduced from e.g. relations (12) and (13) about the ruin amount and portfolio life-time if ruin occurs.

In expression (12) $g_{t}(z)$ is the density function of the first ruin amount $Z_{t}$ at time $t$. The expression

$$
g_{t}^{*}(z)=e^{a \cdot z-b \cdot t} \cdot g_{t}(z) / \sum_{t=1}^{\infty} \int_{0}^{\infty} e^{a \cdot z-b \cdot t} \cdot g_{t}(z) \cdot d z
$$

becomes the conditional density of amount $Z_{t}$ at $t$, (under the hypothesis that the ruin occurs) which takes into consideration the size of the ruin (by the factor $e^{a z}$ ) and the distance in time of the occurrence of the ruin (by the factor $e^{-b \cdot t}$ ).

Let us define

$$
E^{*}(Z \mid T<\infty)=\sum_{t=1}^{\infty} \int_{0}^{\infty} z \cdot g_{t}^{*}(z) \cdot d z
$$

and

$$
E^{*}(T \mid T<\infty)=\sum_{t=1}^{\infty} t \int_{0}^{\infty} g_{i}^{*}(z) \cdot d z
$$

as the "mathematical expectations" of, respectively, the first ruin, amount $Z$ and the portfolio life-time $T$, if ruin occurs, calculated with the modified densities $g_{t}^{*}(z)$. 
These two mathematical expectations are related!

Indeed, expressions (12) and (13) lead to the equality (18)

$$
e^{-a \cdot R_{0}}=\sum_{t=1}^{\infty} e^{-b \cdot t} \int_{0}^{\infty} e^{a \cdot z} g_{t}(z) d z .
$$

By logarithmic derivation with respect to $a$ of the last equation (we are reminded that $b$ is related to $a$ by (9)), we have, after some elementary algebraic simplifications:

$$
-R_{0}=-b^{\prime}(a) \cdot E^{*}(T \mid T<\infty)+E^{*}(Z \mid T<\infty) .
$$

By also taking the logarithmic derivative of (9) with respect to $a$, we find that

$$
P=[\ln M(a)]^{\prime}-b^{\prime}(a)
$$

that is

$$
b^{\prime}(a)=[\ln M(a)]^{\prime}-P .
$$

The first term of the right-hand expression is in fact

$$
[\ln M(a)]^{\prime}=\frac{M^{\prime}(a)}{M(a)}=\frac{\int x \cdot e^{a x} \cdot f(x) \cdot d x}{\int e^{a x} \cdot f(x) \cdot d x}=P_{E}
$$

which is equal to the Esscher premium corresponding to the aggregate claim amount $X$. Thus

$$
b^{\prime}=P_{E}-P .
$$

Relation (19) becomes therefore

$$
-R_{0}=-\left(P_{E}-P\right) \cdot E^{*}(T \mid T<\infty)+E^{*}(Z \mid T<\infty)
$$

or

$$
R_{0}+E^{*}(Z \mid T<\infty)=\left(P_{E}-P\right) \cdot E^{*}(T \mid T<\infty)
$$

This formula can be interpreted as follows: left-hand expression: $R_{0}+$ $E^{*}(Z \mid T<\infty)$ is, at the time of ruin, the average total loss of the company; right-hand expression: $\left(P_{E}-P\right) . E^{*}(T \mid T<\infty)$ is, at the time of ruin, the average deficit in premiums in respect to the level of the Esscher premium and accumulated during the portfolio's life-time. It is to be noted that these are not average values in the usual statistical sense, but averages in the sense of the counter-utility theory, by means of the modified densities $g_{t}^{*}(z)$ which take into account the phenomena of risk aversion and time-stumping. That a relation should exist between the company's total loss and the deficit in premium is not unnatural. It is perhaps surprising that this relation is that simple.

In practice it is clear that it is not at all easy to calculate the expectations $E^{*}(Z \mid T<\infty)$ and $E^{*}(T \mid T<\infty)$. Formula (21) allows at least an estimation of 
one if there is a hint of the value of the other. It seems that the estimation of $E^{*}(T \mid T<\infty)$ is less tricky than that of $E^{*}(Z \mid T<\infty)$ to which many authors have applied themselves.

\section{A NUMERICAL EXAMPLE}

Given a portfolio with the following characteristics:

Risk $X$ (millions of francs)

\begin{tabular}{ccc}
$\begin{array}{c}\text { Claim Amount } \\
1 \text { year } \\
x\end{array}$ & $\operatorname{Prob}(X=x)$ & \\
\cline { 1 - 2 } 90 & 0.1 & \\
90 & 0.2 & $E(X)=100$ \\
100 & 0.4 & $\operatorname{Var}(X)=120$ \\
110 & 0.2 & \\
120 & 0.1 & \\
$M(a)=\frac{1}{10}\left(e^{80 a}+2 e^{90 a}+4 e^{100 a}+2 e^{110 a}+e^{120 a}\right)$.
\end{tabular}

\section{Finance}

Risk premium $\quad P=110$

Initial risk reserve $R_{0}=25$

\section{Ruin}

In the present example the annual surplus can only take values which are multiples of 10 , and the initial risk reserve is 25 , so that an eventual ruin amount will always be: $Z=z_{0}=5$. In order to simplify, we will designate by $q_{t}$ the probability of the first ruin at $T$ :

$$
\int_{0}^{\infty} g_{t}(z) d z=q_{t}
$$

\section{Probability of the First Ruin}

A direct calculation, by repeated convolutions, gives the following values for the probabilities of the first ruin for $t=1,2,3, \ldots$.

The long-term ruin probability $\psi$ is

$$
\psi=\sum_{t=1}^{\infty} q_{t}=0.002446
$$


TABLE 1

RUIN PROBABILITIES

\begin{tabular}{|c|c|c|c|c|}
\hline \multirow[b]{2}{*}{$\begin{array}{r}t \\
(1)\end{array}$} & \multicolumn{2}{|c|}{ Ruin Probabilities } & \multicolumn{2}{|c|}{$\begin{array}{c}\text { Conditional } \\
\text { Ruin probabilities }\end{array}$} \\
\hline & $q_{t}$ & $\begin{array}{c}\text { Accumulated } \\
\text { (3) }\end{array}$ & $\frac{q_{t}}{\sum q_{t}}=q_{t}^{*}$ & $\begin{array}{c}\text { Accumulated } \\
\text { (5) }\end{array}$ \\
\hline 1 & 0.000000 & 0.000000 & 0 & 0 \\
\hline 2 & 0.000000 & 0.000000 & 0 & 0 \\
\hline 3 & 0.001000 & 0.001000 & 0.4088 & 0.4088 \\
\hline 4 & 0.000600 & 0.001600 & 0.2453 & 0.6541 \\
\hline 5 & 0.000360 & 0.001960 & 0.1472 & 0.8013 \\
\hline 6 & 0.000206 & 0.002166 & 0.0842 & 0.8855 \\
\hline 7 & 0.000118 & 0.002284 & 0.0482 & 0.9337 \\
\hline 8 & 0.000068 & 0.002352 & 0.0278 & 0.9615 \\
\hline 9 & 0.000039 & 0.002391 & 0.0160 & 0.9775 \\
\hline 10 & 0.000023 & 0.002414 & 0.0094 & 0.9869 \\
\hline 11 & 0.000013 & 0.002427 & 0.0053 & 0.9922 \\
\hline 12 & 0.000008 & 0.002435 & 0.0033 & 0.9955 \\
\hline 13 & 0.000005 & 0.002440 & 0.0020 & 0.9975 \\
\hline 14 & 0.000003 & 0.002443 & 0.0013 & 0.9988 \\
\hline 15 & 0.000002 & 0.002445 & 0.0008 & 0.9996 \\
\hline 16 & 0.000001 & 0.002446 & 0.0004 & 1.0000 \\
\hline 17 & 0 & 0.002446 & 0 & 1.0000 \\
\hline
\end{tabular}

First Case: Classical Theory, Infinite Time Horizon

Premium $P=110$ and risk $X$ are equivalent in counter-utility, in the sense of relation (1), for $a=0.2004494$. According to (8), ruin counter-utility $\bar{U}(Z)$, risk counter-utility $\bar{U}\left(\overline{\boldsymbol{R}}_{t}\right)$ and Lundberg's upper bound of the ruin probability are identical

$$
\bar{U}(Z)=\bar{U}\left(\bar{R}_{t}\right)=e^{-a R_{0}}=0.006663 .
$$

As the ruin amount is constant by nature $\left(Z=z_{0}=5\right)$, the integral in (7) can be written because of $(22)$

$$
\int_{0}^{\infty} e^{a z} \cdot g_{t}(z) \cdot d z=e^{a \cdot z_{0}} \int_{0}^{\infty} g_{t}(z) \cdot d z=e^{a z_{0}} \cdot q_{t} .
$$

Expression (7) therefore becomes

$$
\bar{U}(Z)=e^{a \cdot z_{0}} \cdot \sum_{t=1}^{\infty} q_{t}
$$

from which we can conclude that

$$
\sum_{t=1}^{\infty} q_{t}=\frac{\bar{U}(Z)}{e^{a z_{0}}}=\frac{e^{-a \cdot R_{0}}}{e^{a \cdot z_{0}}}=\frac{0.006663}{2.724397}=0.002446 .
$$

We find the value obtained by direct calculation, according to (23). 


\section{Second Case: Extended Theory, Finite Time Horizon}

If we fix the horizon $\theta$ (Table 2, first column), the columns (2), (3) and (4) give, respectively, the values of the aversion coefficient $a$, the stumping coefficient $b$ and the stumping factor $e^{-b}$. We find in column (5) the value of the ruin counter-utility, according to the formulae (12) or (13):

TABLE 2

RUIN COUNTER-UTILITIES

\begin{tabular}{rccccc}
\hline \hline $\begin{array}{c}\theta \\
(1)\end{array}$ & $\begin{array}{c}a \\
(2)\end{array}$ & $\begin{array}{c}b \\
(3)\end{array}$ & $\begin{array}{c}e^{-b} \\
(4)\end{array}$ & $\bar{U}(Z)$ \\
$(5)$
\end{tabular}

The above table states that the improvement of the measure $\bar{U}(Z)$ chosen to estimate the financial security of a portfolio is not radical when we bring forward the infinite horizon to a 10 -year horizon, for example; the reduction is more appreciable if we switch to a horizon of 5 or 3 years. This is conform to the known property which states that if ruin occurs, it usually occurs in the near future. A comparison between the ruin probabilities accumulated over a period of $t$ years (table 1 , column 3 ) and the ruin counter-utilities in a horizon of $\theta$ years (table 2, column 5) gives the following:

TABLE 3

COMPARISON BETWEEN RUIN ProBabILITIES AND RUIN COUNTER-UTILITIES

\begin{tabular}{rccc}
\hline $\begin{array}{c}t \\
\text { Years }\end{array}$ & $\begin{array}{c}\text { Accumulated Ruin } \\
\text { Probabilities }\end{array}$ & $\begin{array}{c}\theta \\
\text { Years }\end{array}$ & $\begin{array}{c}\text { Ruin Counter-Utility } \\
\text { with Horizon } \theta\end{array}$ \\
\hline 3 & 0.001000 & 3 & 0.002520 \\
5 & 0.001960 & 5 & 0.003540 \\
10 & 0.002414 & 10 & 0.004747 \\
$\infty$ & 0.002446 & $\infty$ & 0.006663 \\
\hline
\end{tabular}

It can be stated that, for a common period $t=\theta$, the ratios between the two measures of ruin (probability and counter-utility) are rather stable.

Relation (21) between Average Ruin Amount and Average Portfolio Life-Time, if Ruin Occurs

The portfolio under consideration generating constant ruin amounts $\left(Z=z_{0}=5\right)$, the conditional probabilities $g_{t}^{*}(z)$ (in the sense of the counter-utility theory) 
are reduced, for $b=0$ (that is without stumping phenomenon) to the usual conditional probabilities ( $Z$ takes only the value $z_{0}=5$ )

$$
g_{t}^{*}(z)=q_{t} / \sum_{t=1}^{\infty} q_{t}=q_{t}^{*} .
$$

The calculation of $E(T)$ on the basis of the probabilities in Table 1, column 4, gives us

$$
E(T \mid T<\infty)=4.407 .
$$

The direct calculation of the Esscher premium according to (20) gives us

$$
P_{E}=116.803 \text {. }
$$

The relation (21)

$$
R_{0}+E^{*}(Z \mid T<\infty)=\left(P_{E}-P\right) \cdot E^{*}(T \mid T<\infty)
$$

is verified, because

$$
25+5=(116.803-110) \cdot 4.407 \text {. }
$$

\section{REFERENCES}

AMSLER, M.-H. (1975) La Ruine et sa Contre-Utilité. Bulletin of the Association of Swiss Actuaries. Part 2, 167-182.

Gerber, H. U. (1979) An Introduction to Mathematical Risk Theory. Warton School University of Pennsylvania, Chapter 9.

SEAL, H. L. (1978) Survival Probabilities, The Goal of Risk Theory. John Wiley \& Sons: New York. 